\title{
DYNAMIC STRUCTURAL NEURAL NETWORK
}

\author{
$\mathrm{Cu}$ Nguyen Giap ${ }^{1}$, Le Hoang Son ${ }^{2,3 *}$, Francisco Chiclana ${ }^{4}$ \\ ${ }^{1}$ Faculty of Management Information System and E-Commerce, ThuongMai University, Hanoi, Viet Nam \\ cunguyengiap@tmu.edu.vn \\ ${ }^{2}$ Institute of Research and Development, Duy Tan University, Danang, Viet Nam \\ ${ }^{3}$ VNU University of Science, Vietnam National University, Viet Nam \\ sonlh@vnu.edu.vn \\ ${ }^{4}$ Centre for Computational Intelligence, School of Computer Science and Informatics, Faculty of \\ Technology, De Montfort University, Leicester, UK \\ chiclana@dmu.ac.uk
}

*: Corresponding author. Tel.: (84) 904.171.284. Address: 334 Nguyen Trai, Thanh Xuan, Hanoi, Vietnam

\begin{abstract}
Artificial neural network (ANN) has been well applied in pattern recognition, classification and machine learning thanks to its high performance. Most ANNs are designed by a static structure whose weights are trained during a learning process by supervised or unsupervised methods. These training methods require a set of initial weights values, which are normally randomly generated, with different initial sets of weight values leading to different convergent ANNs for the same training set. Dealing with these drawbacks, a trend of dynamic ANN was invoked in the past year. However, they are either too complex or far from practical applications such as in the pathology predictor in binary multi-input multioutput (MIMO) problems, when the role of a symptom is considered as an agent, a pathology predictor's outcome is formed by action of active agents while other agents' activities seem to be ignored or have mirror effects. In this paper, we propose a new dynamic structural ANN for MIMO problems based on the dependency graph, which gives clear cause and result relationships between inputs and outputs. The new ANN has the dynamic structure of hidden layer as a directed graph showing the relation between input, hidden and output nodes. The properties of the new dynamic structural ANN are experienced with a pathology problem and its learning methods' performances are compared on a real well known dataset. The result shows that both approaches for structural learning process improve the quality of ANNs during learning iteration.
\end{abstract}

Keywords: Artificial neural network; Binary multi-input multi-output problems; Dynamic structure; Genetic algorithm; Greedy algorithm; Medical diagnosis. 


\section{INTRODUCTION}

Artificial neural network (ANN) has been applied in numerous areas for a period of time. It has been widely studied and many ANN models have been introduced, which are normally categorized in terms of ANN architectures, activate functions and learning methods [32]. Most ANNs are designed with a static structure whose weights are trained during a learning process by supervised or unsupervised methods [8]. These training methods require a set of initial weights values, which are normally randomly generated, with different initial sets of weight values leading to different convergence for the same training set [7]. Even though ANN is considered a black box that represents a complex functional relationship between outputs and inputs, inside information of relation or cross affection between them is not well comprehended [33].

Let us consider pathology prediction (aka medical diagnosis), which is a binary multi-input multi-output (MIMO) problem, as an example to illustrate why static structure ANN should be enhanced. A remarkable area of application of ANN is pathological expert systems, particularly in predicting health problems of people from their precedent symptoms [6]. In this problem, the appearance or absence of a symptom is represented by a binary variable and the prediction result is a binary variable as well. A feed-forward ANN using back-propagation learning method is often used therein. Besides, Deep Neutral Network [6], probability ANN [27], neuro-fuzzy system [28], etc. have been utilized alternatively. However, these models require a large number of iterations in the training stage and there is no guarantee for its convergence, especially when using short training data and a small error is required [4]. Moreover, traditional learning methods treat each instance of the training dataset the same and therefore the presence of exceptional inputs severely influences the ANN error and training rate [2].

Dealing with these drawbacks, a trend of dynamic ANN was invoked in the past year. The term 'dynamic' here involves the changes on the network's structure or changes on the edges' weights. Vanini et al. [31] proposed using dynamic neural networks for fault detection and isolation in aircraft jet engine. Wu et al. [34] described a novel method called Deep Dynamic Neural Networks for multimodal gesture recognition including a Gaussian-Bernoulli Deep Belief Network to handle skeletal dynamics, and 3D Convolutional Neural Network to manage and fuse batches of depth and RGB images. Jin et al. [14] designed a dynamic neural network for recurrent calculation of manipulability-maximal control actions for redundant manipulators under physical constraints in an inverse-free manner. Aczon et al. [1] proposed a recurrent neural network to learn the course of patient encounters. Amozegar and Khorasani [3] developed an ensemble of dynamic neural network identifiers including a dynamic multi-layer perceptron, a dynamic radial-basis function neural network, and a dynamic support vector machine.

Gaunt et al. [9] accelerated the Dynamic Neural Network through Asynchronous ModelParallel Training. Mustafa, Allen and Appiah [18] used Dynamic Multi-Layer Perceptron to minimize the required computational resource for an effective mobile-based speech recognition system. Torabi et al. [29] proposed a dynamic fuzzy neural network based on sequential fuzzy clustering for fault diagnosis. It uses Adaptive 
Neural Fuzzy Inference Systems (ANFIS) to generate a set of IF-THEN fuzzy rules. After training, a prune process is applied to eliminate neurons that are less important or present redundant fuzzy rules to construct more precise neural network structure. Online dynamic neutral networks, on the other way, focus on online training algorithms.

Pan et al. [22] used both online structure learning and online parameters learning to form an efficient online training progress of ANFIS. They also used an adaptive fuzzy control for online learning progresses [21, 23]. Pratama et al. [24-25] proposed condition monitoring approaches on learning progress, particularly improving two meta-cognitive what-to-learn and when-to-learn. They modified the original pClass by adding a process that initializes new fuzzy rules from an empty set so that a new model called pClass+ has the ability to end up with open and dynamic structure network [26].

Ghiassi et al. [10] proposed a successful dynamic architecture for Artificial Neural Networks called DNN2, in which the hidden layer includes many layers. A layer in the hidden layer has four nodes fixed but the number of layers is dynamically determined. This architecture has been well applied in forecasting time series events [10] and Pre-Production Forecasting of Movie Revenues [11]. Inspired by dynamic structure ANN, Han et al. [12] have introduced a new dynamic structure neural network for adaptive dissolved oxygen control issue. Their approach initializes the hidden layer with zero or few units and updates the hidden layer by adding more units during training process. The other success of using dynamic structure ANN is presented in [16], where the authors developed a modification of Weiner-
Type dynamic ANN for Modeling of Nonlinear Microwave Devices that can be implement in both software and hardware devices. Other works regarding the dynamic neural network can be seen in $[17,19,20]$.

Even though there have been recent advances on the dynamic neural networks, they are either too complex or far from practical applications. For example, a pathology predictor in MIMO problems works like a multi-agent system in which the combination of each separate insiders' activities influences the final prediction result. In fact, the appearance of a symptom itself would not be a clear indication for a specific illness but a set of co-appearance of symptoms. Moreover, within a set of symptoms some of them might not strongly belong to a specific illness; they are just precedents for other symptoms that directly belong to the true diagnosed illness. Capturing this aspect, when the role of a symptom is considered as an agent, a pathology predictor's outcome is formed by action of active agents while other agents' activities seem to be ignored or have mirror effects. The current dynamic neural networks are incapable to model such the cases.

In order to mimic the relationship between agents in a system, we have to think about a new dynamic structural ANN model (e.g. for MIMO) including three layers with the most important different layer being the hidden layer. The hidden layer, which is modeled by the dependency graph, includes the set of nodes within dynamic adaptive topology. It means that the structure of the proposed ANN can represent the relationship between outputs and inputs; and the structure can be learnt or be updated during the learning process. By doing so, the dynamic 
structural ANN is able to handle the variation of input data as well as the limitations above.

In this paper, we propose a new Dynamic ANN for binary MIMO problems based on the dependency graph, which provides clear cause and result relationships between inputs and outputs. The dependency graph has been used in intelligent systems and applications such as instruction scheduling and job shop scheduling because it gives a clear relationship between elements involved in the system [13]. In this study, the dependency graph is applied in order to build a dynamic structural ANN that hierarchy both features of ANN and the dependency graph. In the new model, the dependency graph is used as a hidden layer and its topology is trained during the learning process of ANN.

The rest of paper is organized as follows. Section 2 describes the proposal dynamic structural ANN and several proposed learning methods. Section 3 shows the experimental results on pathology prediction problem. Finally, the main results and further works are discussed in Section 4.

\section{DYNAMIC STRUCTURAL ANN}

In this section, the new dynamic structural ANN will be presented and it will be applied to the pathology prediction problem for illustration purposes. Specifically, Section 2.1 addresses the main ideas of the proposed ANN, including its theoretical validation. Section 2.2 proposes the design of the new dynamic structural ANN, while Section 2.3 focuses on two learning algorithms for the proposed ANN: greedy algorithm and Genetic Algorithm (GA). Lastly, Section 2.4 summarizes the advantages of the proposal against other works.

\subsection{Main ideas}

Considering pathology prediction as a multiagent system, each of its symptoms has a different impact on a body and their insiders' activities influence a prediction result. An agent mimics the action of a symptom or a set of similar effect symptoms. In fact, the appearance of a symptom itself might not give an insight direction for any specific illness because that symptom may belong to many illnesses. This is the case with fever, which is a symptom of wide spread diseases. However, a set of co-appearance symptoms provides a more confident clue of a specific illness. Even though the symptoms of an illness change during the time of such illness, in its middle stage symptoms are stable. In any case, within the set of symptoms, some might not directly belong to an illness; they just are precedents for other symptoms that directly belong to a diagnosed illness. This means that the outcome of pathology prediction is formed by action of active agents while other agents' activities seem to be ignored. This relation would be clearly represented by a dependency graph [13].

The outcome of a dependency graph is not evaluated if there is a circular dependency [13]. Indeed, if no specific order is rationally applied for a circular of dependencies then no object is calculated first. To overcome this for a dependency graph, a directed acyclic graph needs to be formed. Combining features of dependency graph and properties of a real pathology diagnosis, a new dynamic structural ANN is proposed whose structure mimics real role of symptoms in a disease prediction system. The proposed new ANN mimics different effects of an agent to others and different agents' roles to the result. 
In principle, the proposed ANN has three layers: an input layer, a middle layer, and an output layer (Fig. 1). Each agent has different view for input signals and is represented by different input vectors. The output vector shows different roles of an agent in a specific economic factor. The different roles of agents are also represented by connections between agents. Therefore, an active agent effect on others can be represented by high number of nodes connections, while the output vector can be used to represent its effect on the output.

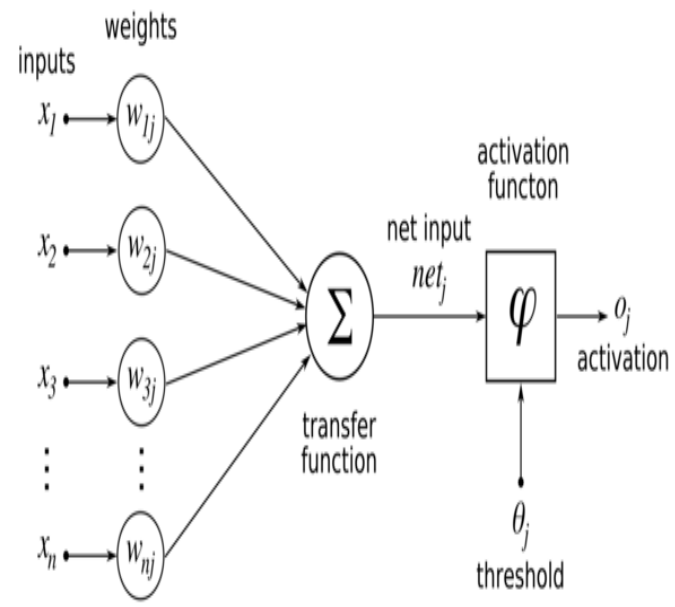

Fig. 1. Architecture of static ANN

In the new dynamic structural ANN, the role of nodes is strongly represented by its topology of connection rather than connections' weights. Therefore, a fixed weight is applied to all edges to avoid the affection of weight in this type of ANN. Certainly, it needs to be proved that the proposed ANN using dynamic topology and a fixed weight works as the original ANN. Fig. 1 depicts the architecture of a traditional static ANN.

The existence of an ANN with a single fixed weight $\bar{w}$ that works as the above architecture is proven by replacing a single input $x_{i} w_{i j}$ in static
ANN by a sub ANN with single fixed weight $\bar{w}$. As all inputs of a node will be replaced by sub ANNs that have fixed weight, a static ANN is transposed into an expected ANN. In this study, such existence is proved only in case of positive activation functions. Notice that the most wellknown and applied activation function for ANN is the sigmoid function, which is a positive function. Thus, we have the following lemma.

Lemma1. If ANN uses a positive activation function, a node can be replaced by a sub ANN having fixed weight.

Proof: Without loss of generality, assume that input $x_{i}$ has corresponding weight $w_{i j}$, which differs from others. We need to build a sub ANN having a single weight $\bar{w}$ for all edges and prove that there exists a structure of sub ANN that has single input $x_{i}$ and the ability to generate same input value $x_{i} w_{i j}$ for node $j$. Denote by $\bar{y}=\varphi\left(x_{i} \bar{w}\right)$ the output of a node having single input $x_{i}$ and weight $\bar{w}$. In the simplest case, the input $x_{i} w_{i j}$ is mimicked by a sub ANN with $\left|x_{i} w_{i j} / \bar{y}\right|$ nodes as illustrated in Fig. 2.

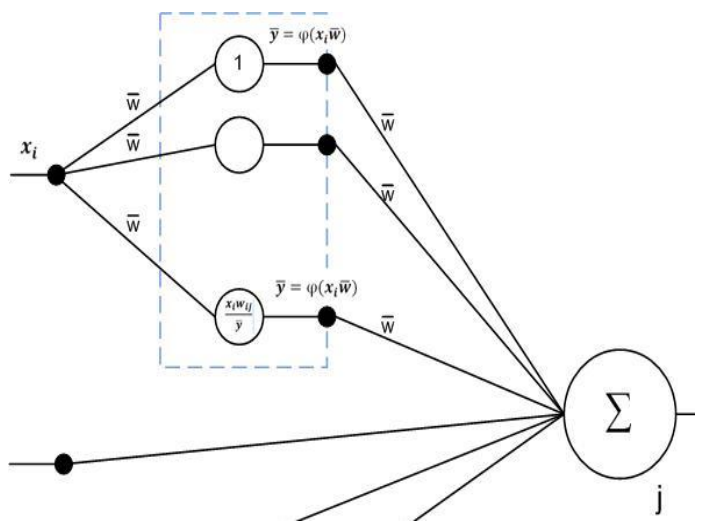

Fig. 2. A neuron with fixed weight 
Node $j$ would be completely replaced by a sub ANN having fixed weight if all its inputs are replaced by the same way above. Therefore, Lemma 1 is proved.

Lemma 2: For each ANN with different weights, there exists an ANN that has fixed weight working the same.

Proof: Replacing the nodes in the ANN with the same structure constructed by Lemma 1 trivially proves this lemma.

However, the question of finding optimal structure of fixed weighted ANN is still open herein. Notice that the sigmoid activation function is both a positive and monotonic function. The positive monotonic property of activation functions gives the key point for searching a suitable topology of dynamic structural ANN. In the ANN using positive monotonic activation functions, the relation between density of structures and output values is clear since the number of connections does not decrease. This is summarized in the following property 1.

Definition 1. Assume two ANNs, A and B, have the same number of nodes and are represented by directed graphs without cycle. Then, B is called a sub ANN of A if all directed edges in B also appear in A.

Property 1. If an ANN uses a positive monotonic activation function, its output value is greater than or equal to those of its sub ANNs.

Proof: We prove that adding an edge to a given ANN does not decrease the output values. Given an ANN A that uses a positive monotonic activation function, without loss of generality, assume that its node $j$ has a new direct edge from node $i$. In this case, the value of $n e t_{j}$ is added, and because the activation function is positive and monotonic the output of node $j$ does not decrease. If the output value of node $j$ is a direct or indirect input of the output node, the output value of A does not decrease. Otherwise, node $j$ does not affect the output node and then the output remains the same. $\square$

Remark 1. Property 1 is a consequence of the following: If B is a sub ANN of A, then A would be formed by adding one or more edges to $\mathrm{B}$ and therefore the output value of $\mathrm{A}$ will be equal to or greater than that of $\mathrm{B}$. There exists a limitation of connection between an acyclic directed graph with a fixed number of nodes. Therefore, a dynamic structural ANN has a limited output value.

Definition 2. A network is called a full connected network if it is represented by a directed graph that has at least one cycle providing that any edge is added into this graph.

Remark 2. In an ANN with specific number of nodes, there is more than one full connected network. This is because once a full connected network is obtained by adding edges to the ANN, the order of adding edges can be changed to produce a new full connected network. For example, in a directed graph, if an edge $(i, j)$ is added, the edge $(j, i)$ cannot be added to avoid cycle, or in an extension, if two edges $(i, j)$ and $(j, k)$ are added then edge $(k, i)$ cannot be added. Therefore, the order of adding edges leads to different full connected network.

Property 2. If an ANN has fixed weight and number of nodes, then output values are upper bounded.

Proof: Starting from a partial network, i.e. a not fully connected network, a full connected network is reached by repeatedly adding an edge 
into the network. As per Property 1, when an edge is added, output values do not decrease. However, with a specific number of nodes, the number of full connected network is limited. Therefore, there exits an upper bound for outputs values and this is generated by the full connected network having highest output values.

Remark 3. In order to break its upper bound, a full connected network must increase the number of nodes. However, in a full connected network, no more edges can be added. Therefore, the only way to break through the upper bound of output values is increasing the number of node. As soon as new nodes are added into a dynamic structural ANN, there is a set of new edges that can be added to increase the output values.

\subsection{Design of the new ANN}

From the above idea, we propose a new ANN that shares several important properties with the original ANN: the topology of a neuron; and three layers architecture. However, a dynamic structural ANN has the following key differences: every connection between nodes in the ANN has the same weight; and the hidden layer is represented by an acyclic directed graph. Each neuron of a dynamic structural ANN has multiple inputs, a fixed weight $w$ and one output with a bias, as depicted in Fig. 3 .

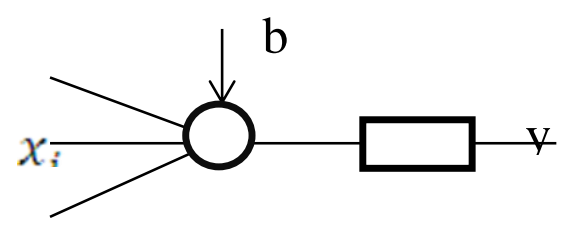

Fig. 3. A neuron of dynamic structural ANN

The activation function is sigmoid as defined below:

$$
\varphi(\text { net })=\frac{1}{1+e^{-n e t}}
$$

The network architecture has three layers: an input layer, a hidden layer and an output layer (Fig. 4):

Input layer: Each input node has connections to hidden nodes; at least one connection represented by an input vector $V_{i}$ has the same weight $w$.

\section{Dynamic hidden layer:}

- One hidden node can connect to any node in the same layer. The number of connections of a node shows its effect on others and, therefore, its indirectly effect on the output.

- A hidden node having direct connection to an output $j$ is an active node of $j$. A hidden node that does not have direct connection to an output $j$ is regarded as a normal/inactive node of $j$.

- All connections have same weights.

- The hidden layer's structure shows the roles of nodes and can be represented by a directed graph. If a node $\mathrm{A}$ has a directed connection to node $\mathrm{B}$, this means that output of node $\mathrm{A}$ is an input of node $B$.

- There is no cycle in a directed graph that represents the hidden layer.

\section{Output layer:}

- The output value depends on the active hidden nodes' output.

- An output vector $U_{j}$ represents the effect of active nodes on the output $j$, and has the same weight.

\section{Output calculation:}

- Outputs of NN are calculated through the activation function and the directed graph of the hidden layer. As described 
in the design, the structure of the hidden layer aims to avoid any directed cycle because a cycle will prevent the calculation process of outputs. In general, the structure of the hidden layer must be represented by an acyclic directed graph.

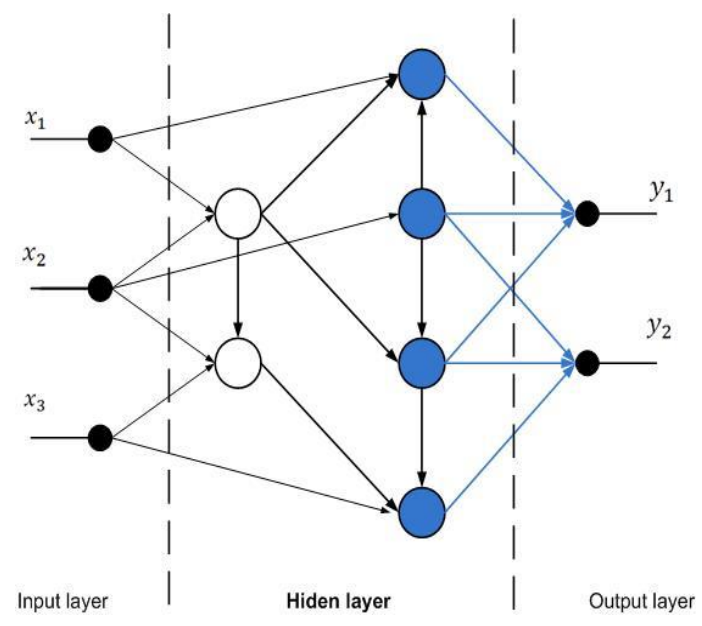

Fig. 4. Architecture of the proposal ANN

\subsection{Learning algorithms}

When applying the new dynamic structural ANN to a real problem, the input and output layers assign its numbers of nodes according to the requirement of the problem at hand. The target of the structural learning process of the new dynamic structural ANN reduces thus to learning the structure of the hidden layer. A directed graph form the structure of all layers represents the number of nodes and their connections. Therefore, the leaning algorithm has to learn a suitable number of nodes and the topological connections for a training data set. Learning the structure of ANN is a new learning process without clear previous instructions [4].

In this section, we propose two approaches for structural learning. The first one learns directly by calculating output errors and uses these errors to update the number of active hidden nodes based on the Greedy algorithm [5]. Then, back propagation process is applied to calculate the active hidden nodes, output errors and update its income connections. This process repeats until an expected error or maximal iteration is reached. The second approach uses Genetic Algorithm (GA) as a key of structural learning. A set of ANN structures is initialized as a population, and then using the output errors as fitness function. The GA will lead to the best possible structure of the ANN even if it is not a global optimal structure.

\subsubsection{Greedy algorithm}

The structure of the hidden layer is learnt using Property 1 and Property 2: increasing the number of connections or active nodes in the hidden layer when using a positive monotonic activation function leads to increasing the output values. However, as the network structure forms a full connection network and a new connection cannot be added, the only option, according to Property 2 , is to add a new node. The priority between the two above factors determines the final resultant structure with high number of nodes. Therefore, in this section we use the approach with priority of topological connections. The designed learning method has two stages. The first stage initializes the parameters of the ANN, and the second stage learns the hidden layer structure by input data stream.

\section{Stage one}

- Randomly initialize a number of hidden nodes $N$ in the interval [2n-MAX_INT] where $n$ is the number of inputs, $M A X \_I N T$ is the maximum value for a 


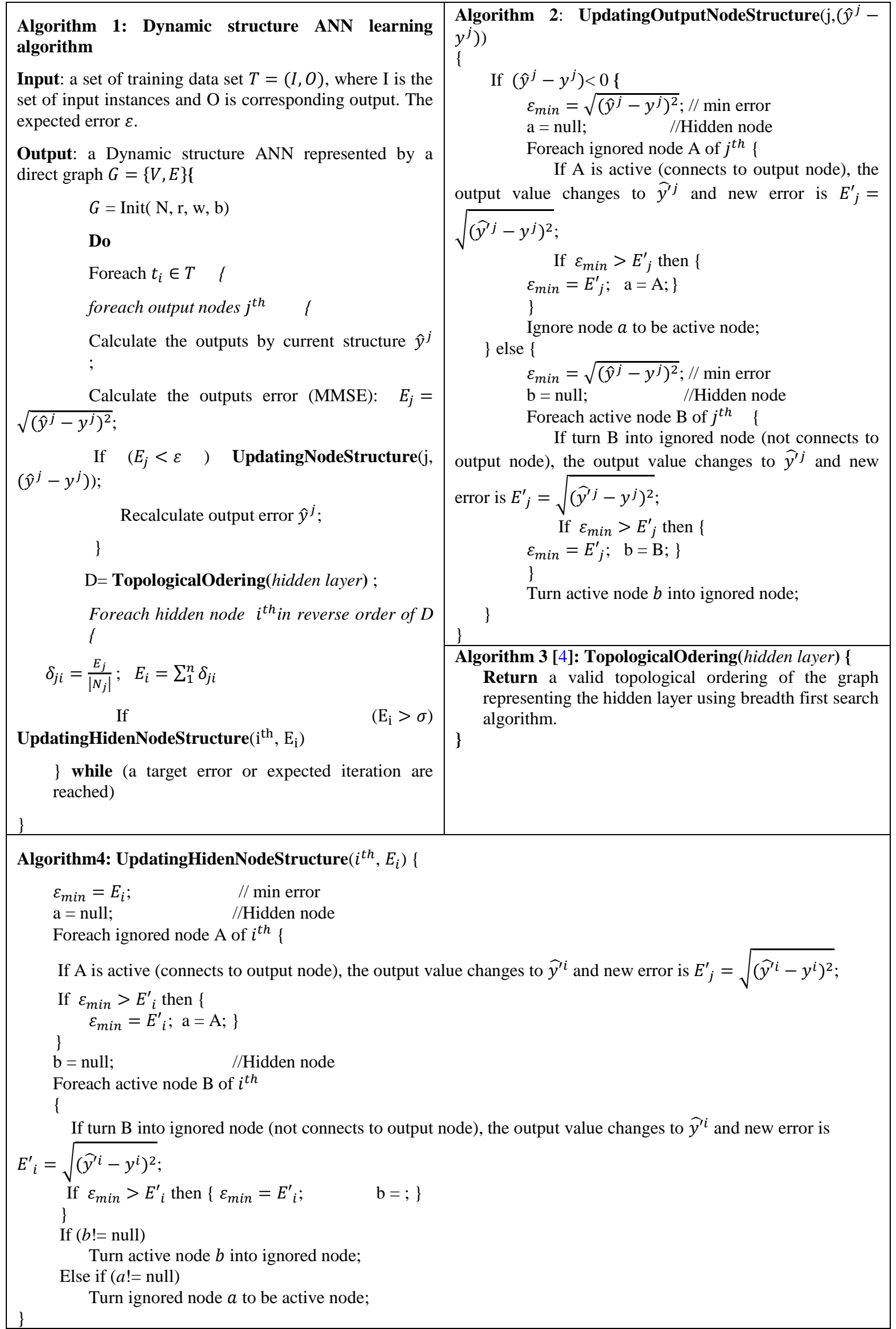


32 bit integer and a structure of hidden layer (with small number of connections). The number of connections in the initial structure should be small in order to capture the basic information from patterns. A tuple of bias and weight is also important in this structural learning, and they are set by the ANN designer [15]. The bias is set as high enough, much higher than the weight, to ensure that the number of hidden nodes required for a specific problem is acceptable [15].

\section{Stage two: structural learning process}

- In general, minimizing the error of a trained structural $\mathrm{ANN}$ on a specific training data set is the target of the structural ANNs' learning algorithm. In case of fixed-weight dynamic structural ANNs, the learning process has to change the connection between nodes for better outcome according to Property 1.

- This stage points out that in an iteration of learning only active nodes are learnt directly under the output errors [15]. And then, the active nodes request the input ones to learn.

Algorithms 1-4 change the connections to minimize the output errors. The learning algorithm based on greedy algorithm has rate of convergence dependent on the characteristic of a problem. However in one iteration, the learning algorithm updates all output nodes and continues the update of relevant active nodes in the hidden layer. The number of active nodes is Smaller than or equal to the total number of nodes of the hidden layer. Updating an active node in worst case has to update all nodes in the hidden layer.
Update one node is simple task by turning a selection connection on or off. Therefore, the complexity of the learning algorithm is $\mathrm{O}(N,|Y|)=\left(|Y| \cdot N^{2}\right)$ where $\mathrm{N}$ is the number of hidden nodes and $|Y|$ is the number of output nodes.

\subsubsection{Genetic algorithm}

This section uses GA algorithm for learning the structure of the dynamic structural ANN. In general, the GA algorithm has four stages. The first stage initializes a population of problem solutions. The three remaining stages are selection, crossover and mutation, which are repeated until the population converges or a fixed number of iterations is reached [5]. In order to use GA algorithm, the first task is to represent the problem as a chromosome. The structure indeed includes the number of hidden nodes, the connections between them and the connections between hidden nodes and output nodes. Assume the number of input nodes is $l$, the number of hidden nodes is $n$ and the number of output nodes is $m$. The structure of the ANN is represented by a binary matrix of dimension $(n) *(l+n+m)$. Each row $i^{t h}$ of the matrix represents the out connections of a hidden node $i^{\text {th }}$ to other hidden nodes and input, output nodes. A chromosome to represent the above structure matrix is a chain with $(n) *(l+n+$ $m$ ) bits [5].

However, this representation of a chromosome would not guarantee that crossover generates two new valuable chromosomes for dynamic structural ANN. The fact is that, a chromosome must represent an acyclic directed graph, but the crossover of two chromosomes would generate new chromosomes that represent 
a directed graph having cycle. Therefore, a modification of crossover is introduced here for dynamic structural ANN. In an acyclic directed graph, there is an order of nodes and the connections are fixed from lower rank node to higher rank node. Therefore, in order to use GA, all chromosomes have the same order of nodes, which can be set as $\{1,2, \ldots, n\}$ without loss of generality. New modification of crossover for dynamic structural ANN has to keep the order of nodes to guarantee that new chromosomes are still acyclic directed graph. Subject to this constraint, the crossover process has to choose the cut point at the end of the nodes in a chromosome. In this case, as two chromosomes

\begin{tabular}{|c|c|}
\hline \multicolumn{2}{|c|}{ Algorithm 5: Genetic Algorithm } \\
\hline \multicolumn{2}{|c|}{$\begin{array}{l}\text { Input: a set of training data set } T=(I, O) \text {, where } I \text { is } \\
\text { the set of input instances and } O \text { is corresponding } \\
\text { output. } \\
\text { Output: a Dynamic structure ANN represented by a } \\
\text { direct graph } G=\{V, E\} \\
\{ \\
\text { Initialization }\end{array}$} \\
\hline - & $\begin{array}{l}\text { Select a number of populations in } \\
\text { initial generation ( } \mathrm{N} \text {, select a fix } \\
\text { number of hidden nodes } n \text {, and } \\
\text { randomly choose an order } \\
D=\left\{v_{i}\right\} \text { of } n \text { nodes. } \\
\text { For each initial structure: } \\
\text { Randomly create a } \\
\text { structure of hidden layer } \\
\text { by randomly choose a } \\
\text { number } z \text { of connection. } \\
\text { Repeats } z \text { time: choose } \\
\text { randomly two hidden } \\
\text { nodes } A, B \text { and create a } \\
\text { connection from } A \text { to } B, \\
\text { ensure that A stand } \\
\text { before B in order } D \text {. }\end{array}$ \\
\hline \multicolumn{2}{|c|}{$\begin{array}{l}\text { While (the error of the best chromosome in current } \\
\text { population larger than target of number of iteration } \\
\text { smaller than expected number) }\end{array}$} \\
\hline \multicolumn{2}{|l|}{ f } \\
\hline \multicolumn{2}{|l|}{ Selection } \\
\hline & $\begin{array}{l}\text { Calculate fitness function of all } \\
\text { chromosomes in current } \\
\text { population. } \\
\text { Using tournament selection } \\
\text { mechanism to select parents set. } \\
\text { Repeats } \mathrm{N} \text { time the following }\end{array}$ \\
\hline
\end{tabular}

exchange their part, the new chromosomes represent two acyclic directed graphs with the same order of nodes. The purpose of GA is finding the network structure that has smallest error on the training data set. Therefore a fitness value of a network is estimated by the below equation (2) where $\partial_{j k}$ is the error of output $j$ for an instance of inputs $k$. This fitness function satisfies the requirements of GA: the value of fitness function is positive and this value increases when the fitness of solution increases.

$$
\text { fitness }=\frac{1}{\sum_{k=1}^{m} \sum_{j=1}^{n} \partial_{j k}}
$$

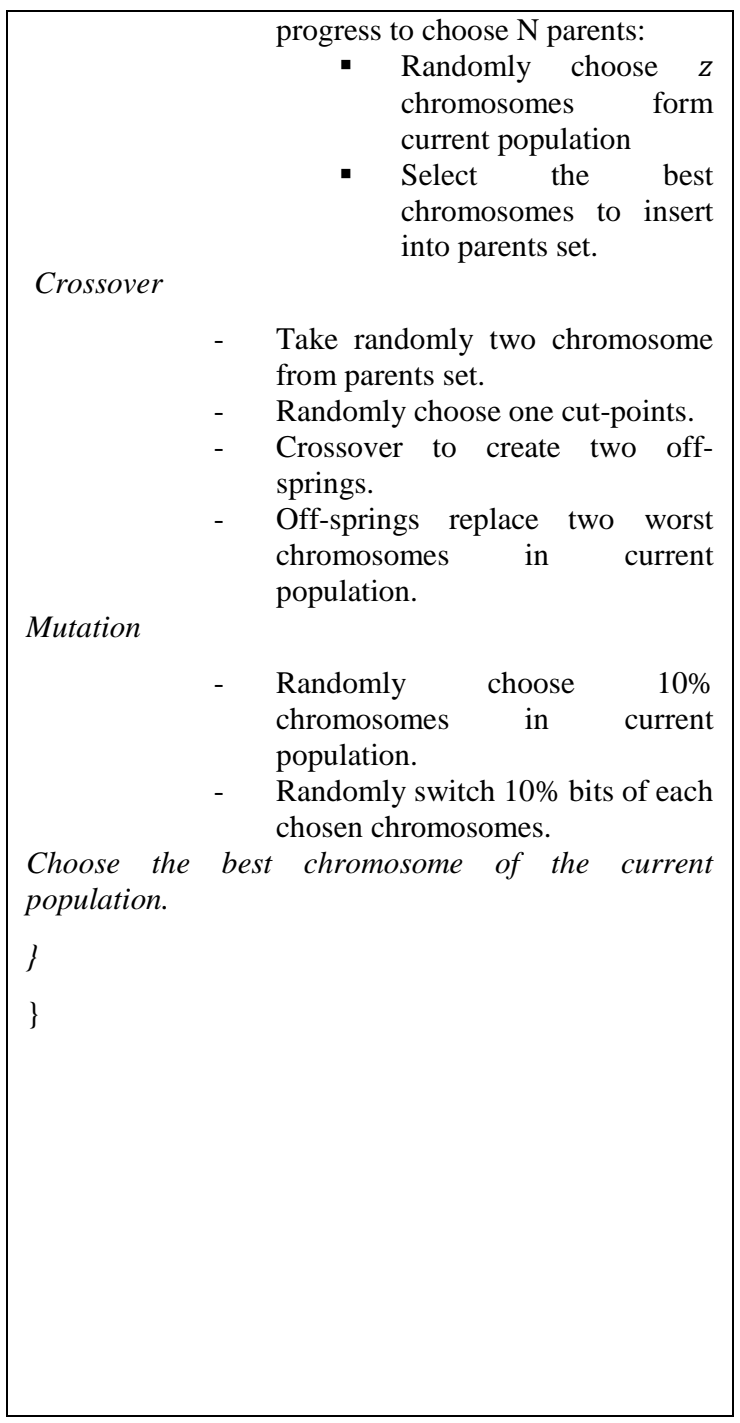


There are several remarkable advantages of the designed GA for NN structure learning problem. First, the simplicity of chromosome encryption and the selection, crossover and mutation mechanisms lead to easy implementation. Second, the underneath mathematical process of GAs guarantees to reach local optimal solution. Third, if an initialized population of GAs is large enough and diverse, the final solutions of NN structure will be close to the global optimum.

However, the most important disadvantage of $\mathrm{GA}$ is computing cost. GA requires calculating fitness values for all chromosomes of each generation, and these values are estimated for all input instances of training set making it computationally expensive. Reducing the number of chromosomes or the number of generations will not solve this issue because they directly influence to quality of GA. However the computation time of GA can be reduced by using Parallel GA [5]. The second disadvantage of GA is that it works as a "gray-box", meaning that although the GA has a clear reaching target, such as the fitness of a structure for a specific input set, it does not show the relationship between fitness convergence and structural change. In other words, the algorithm does not have a specific method to change the NN structure based on target error. The process of GA algorithm for the dynamic structural ANN is depicted in Algorithm 5.

In the learning method based on GA, the rate of convergence is depended on the characteristics of a problem where it is applied. However, the complexity of algorithm in one iteration of GA depends on selection, crossover and mutation processes.

\subsection{Advantages of the proposal}

The proposed dynamic structure ANN differs from other dynamic ANNs mentioned in the surveyed literature by using a dependency graph to mapping between multi-inputs and multi-outputs. In the scope of this paper, we used fixed weights for any connections between nodes of the three layers: input layer, hidden layer and output layer. This approach turns the dynamic structure into the center of research, where it has also been proved that a suitable dynamic would be constructed by a suitable learning process.

Two learning algorithms are used in the new ANN including the greedy and genetic algorithm approaches; however in future, new other efficient learning processes could be implemented.

The capacity of the proposed dynamic structure ANN depends on the number of nodes of the hidden layer that a computing system can process. Ideally, this number will be extended as the system need (the number of hidden node will increase as the size and diversity of data increase), but in practice, system designers will have to balance computing time with the number of hidden units.

\section{EVALUATION}

This section reports on the experimental results of the proposed dynamic structural ANN on training data of heart attack from UCI [30]. The data has 1 output and 22 inputs; the 22 inputs represent the symptoms of patients and the output is the conclusion about their heart problem. The training set has 80 instances and the testing set has 187 instances. In order to validate the properties of the proposed dynamic structural ANN, we used the two learning methods separately and then compare their accuracy. At first, each learning methods' performance is tested on the training database to 
figure out the best parameters, and then, the results of the two algorithms are compared.

\subsection{Greedy algorithm based learning method}

For running the test of greedy algorithm based learning method, we use different sets of parameter $(\mathrm{N}, \mathrm{r}, \mathrm{w}, \mathrm{b})$ where $\mathrm{N}$ is the number of hidden node, $\mathrm{r}$ is the ratio of initial connections, $\mathrm{w}$ is the weight of connection and $\mathrm{b}$ is the bias of neuron. However, for the purpose of showing the inside properties of the learning method, we have run the test with $\mathrm{N}=100, \mathrm{r}=5 \%, \mathrm{w}=0.05, \mathrm{~b}=0.1$. Each training process runs a maximum of 100 iterations, and the result is estimated by 5 time testing. The training shows several interesting results as below.

\section{Instance error during one iteration:}

During the iterations of the learning process, the error of each instance in the training set varies to balance average errors for all instances. For example, in the first three iterations, the errors of training input are shown in Fig.5.

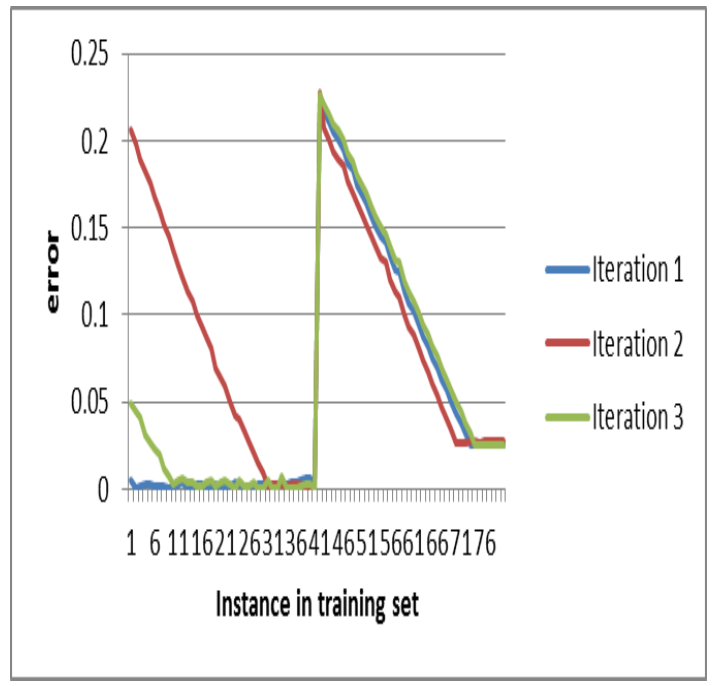

Fig. 5. Learning method performance

\section{Average error vs. number of iteration:}

The result shows that the greedy algorithm, even though designed to improve the quality for each instance in the training set separately, does not guarantee convergence to global optimum. The different instance has affected the ANNs' structure in deferent ways and a structural updating improves for one input instance but reduces results of others (Fig.6).

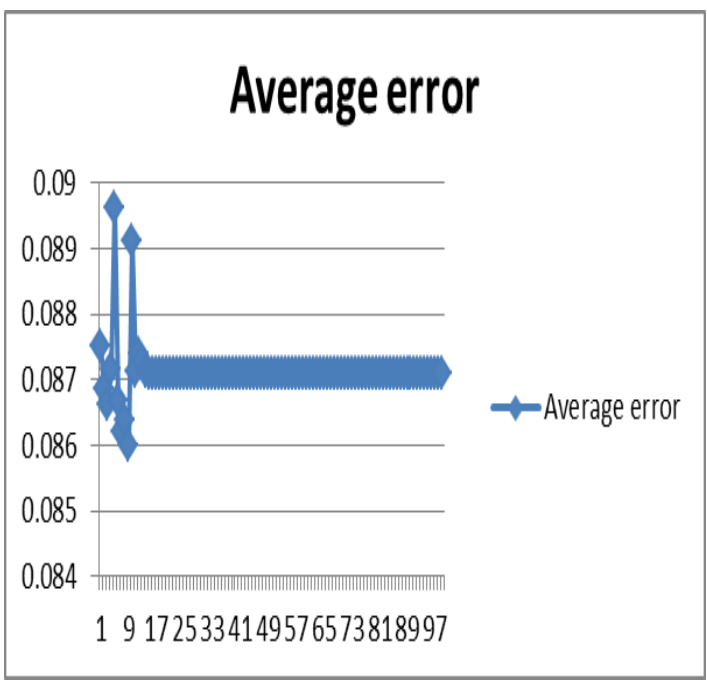

Fig. 6. Average error during training iteration

\subsection{Genetic algorithm based learning method}

We applied GA algorithm with the same number of hidden node tested by the greedy algorithm. In general, GA has several important parameters to test and choose the good candidate. The number of candidates in tournament of selection stage is set at $10 \%$ of the population. The rate of mutation is set at $10 \%$ of connection in a chromosome. The performance of the GA based learning method is shown in Figs. 7-8. In experience, the algorithm leads to local convergence after a short number of iterations. This situation can be improved by increasing the population size, because a larger population gives a more diversity generation, and then the GA will search in larger space, or increasing the mutation rate [5]. The result shows the problem of convergence in GA using tournament selection, with the best chromosome 
having high possibility to reproduce in next generation. Besides, we have to limit the number of cross point selection in the crossover stage; therefore the searching space is limited. The consequence is that the GA converges fast to a local optimum.

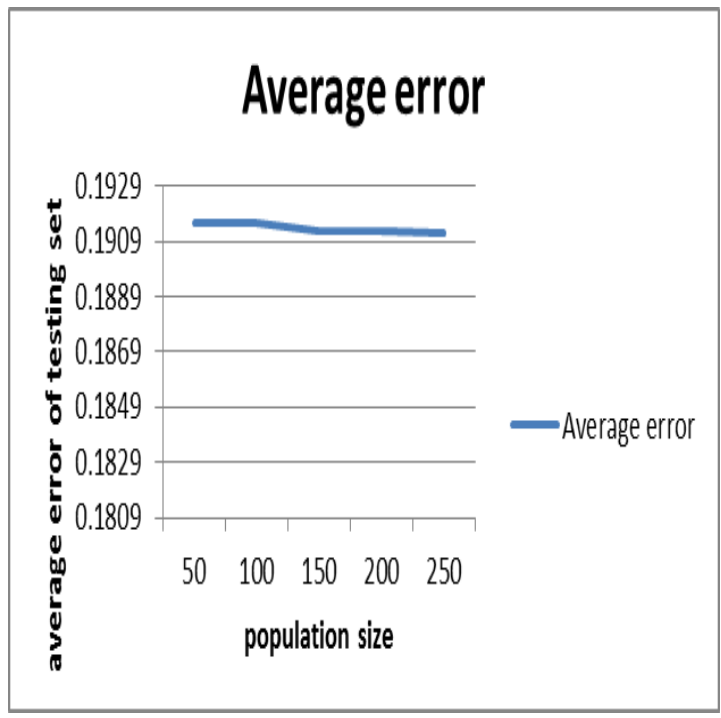

Fig. 7. Average testing error vs. number of population

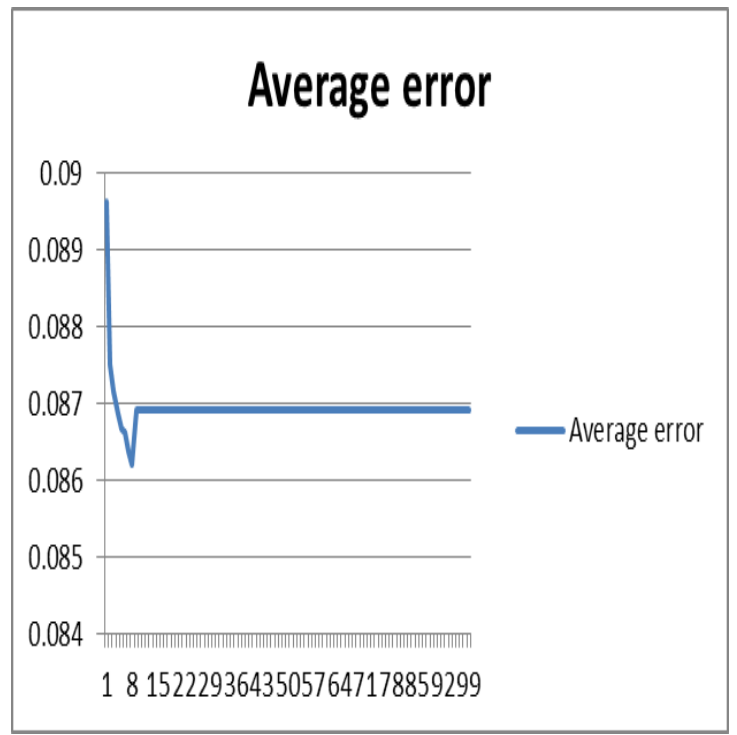

Fig. 8. Average error in training iteration

\subsection{The comparison of learning methods}

In case of using 100 hidden nodes, the performance of both learning methods in the testing set is depicted below (Fig. 9). The experimental results show that GA algorithm gives better prediction for most cases in the testing set.

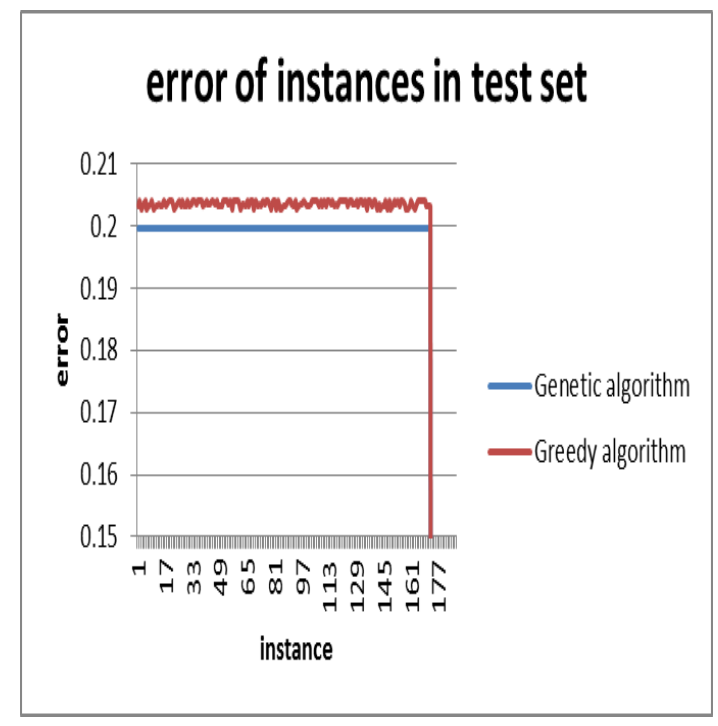

Fig. 9. Two learning methods' performance

\section{CONCLUSIONS}

In this paper, we focused on designing a novel dynamic structural artificial neural network (ANN) for binary multi-input multioutput (MIMO) problems. We introduced the fundamental knowledge for the dynamic structural ANN and its comparison with respect to the static structural ANN. The important definitions and properties of the dynamic structural ANN regarding positive activation functions, such as sigmoid functions, have been theoretically examined. Considering as the first effort to present the dynamic structural ANN for binary MIMO problems, we believe that it is valuable in reality thanks to the dynamically relationship between inputs and outputs with the support of dependency graph. This fosters the adaptability and survival capability of the proposal for a wide variety of practical problems.

The proposed model of dynamic structural ANN has been validated using a real data set of heart attack in UCI. The result shows that both approaches for structural learning process 
improve the quality of ANNs during the learning iteration. In experience, the drawback of both learning methods is caused by the limitation of computing power, which curbs for running test with large number of hidden nodes.

In future, we will expand this research with other learning methods to construct better results for the dynamic structural ANN. Large number of hidden nodes will be tested for better performance, and suitable areas for applications of the new dynamic structural ANN to benefit from the cause and result relationship represented by the ANN's structure will be studied.

\section{ACKNOWLEDGEMENT}

This research is funded by Graduate University of Science and Technology under grant number GUST.STS.ĐT2017- TT02.

The authors are grateful for the support from the Institute of Information Technology, Vietnam Academy of Science and Technology. We received the necessary devices as experiment tools to implement proposed method.

\section{REFERENCES}

1. Aczon, M., Ledbetter, D., Ho, L., Gunny, A., Flynn, A., Williams, J., \& Wetzel, R. (2017). Dynamic Mortality Risk Predictions in Pediatric Critical Care Using Recurrent Neural Networks. arXiv preprint arXiv:1701.06675.

2. Amato, F., López, A., Peña-Méndez, E. M., Vaňhara, P., Hampl, A., \& Havel, J. (2013). Artificial neural networks in medical diagnosis. Journal of applied biomedicine, 11(2), pp.47-58.

3. Amozegar, M., \& Khorasani, K. (2016). An ensemble of dynamic neural network identifiers for fault detection and isolation of gas turbine engines. Neural Networks, 76, 106-121.

4. Azar, A. T. (2013). Fast neural network learning algorithms for medical applications. Neural Computing and Applications, 23(3-4), 1019-1034.

5. Cantú-Paz, E. (2007). Parameter setting in parallel genetic algorithms. Parameter setting in evolutionary algorithms, 259-276.

6. Cireşan, D. C., Giusti, A., Gambardella, L. M., \& Schmidhuber, J. (2013, September). Mitosis detection in breast cancer histology images with deep neural networks. In International Conference on Medical Image Computing and Computer-assisted Intervention (pp. 411-418). Springer, Berlin, Heidelberg.

7. Das, R. (2010). A comparison of multiple classification methods for diagnosis of Parkinson disease. Expert Systems with Applications, 37(2), 1568-1572.

8. Er, M. J., Zhou, M. J. (2008). Automatic generation of fuzzy inference systems via unsupervised learning. Neural Networks, vol. 21, no. 10, pp. 1556-1566.

9. Gaunt, A., Johnson, M., Riechert, M., Tarlow, D., Tomioka, R., Vytiniotis, D., \& Webster, S. (2017). AMPNet: Asynchronous Model-Parallel Training for Dynamic Neural Networks. arXiv preprint arXiv:1705.09786

10. Ghiassi, M., \& Nangoy, S. (2009). A dynamic artificial neural network model for forecasting nonlinear processes. Computers \& Industrial Engineering, 57(1), 287-297.

11. Ghiassi, M., Lio, D., \& Moon, B. (2015). Pre-production forecasting of movie revenues with a dynamic artificial neural network. Expert Systems with Applications, 42(6), 3176-3193. 
12. Han, H. G., \& Qiao, J. F. (2011). Adaptive dissolved oxygen control based on dynamic structure neural network. Applied Soft Computing, 11(4), 3812-3820.

13. He, M., \& Zhang, J. (2011). A dependency graph approach for fault detection and localization towards secure smart grid. IEEE Transactions on Smart Grid, 2(2), 342-351.

14. Jin, L., Li, S., La, H. M., \& Luo, X. (2017). Manipulability optimization of redundant manipulators using dynamic neural networks. IEEE Transactions on Industrial Electronics, 64(6), 4710 - 4720.

15. Keysers, C., \& Gazzola, V. (2014). Hebbian learning and predictive mirror neurons for actions, sensations and emotions. Phil. Trans. R. Soc. B, 369(1644), 20130175.

16. Liu, W., Na, W., Zhu, L., Ma, J., \& Zhang, Q. J. (2017). A Wiener-Type Dynamic Neural Network Approach to the Modeling of Nonlinear Microwave Devices. IEEE Transactions on Microwave Theory and Techniques.

17. Mohammed, A. M., \& Li, S. (2016). Dynamic neural networks for kinematic redundancy resolution of parallel Stewart platforms. IEEE transactions on cybernetics, 46(7), 1538-1550.

18. Mustafa, M. K., Allen, T., \& Appiah, K. (2017). A comparative review of dynamic neural networks and hidden Markov model methods for mobile on-device speech recognition. Neural Computing and Applications, 1-9.

19. Nanda, T., Sahoo, B., Beria, H., \& Chatterjee, C. (2016). A wavelet-based nonlinear autoregressive with exogenous inputs (WNARX) dynamic neural network model for real-time flood forecasting using satellite-based rainfall products. Journal of Hydrology, 539, 57-73.

20. Neubig, G., Dyer, C., Goldberg, Y., Matthews, A., Ammar, W., Anastasopoulos, A., ... \& Duh, K. (2017). DyNet: The Dynamic Neural Network Toolkit. arXiv preprint arXiv:1701.03980.

21. Pan, Y., Er, M. J., Huang, D., Wang, Q. (2011). Adaptive fuzzy control with guaranteed convergence of optimal approximation error.IEEE Transactions on Fuzzy Systems, 19 (5), 807-818.

22. Pan, Y., Er, M. J., Li, X., Yu, H., Rafael, G. (2014). Machine health condition prediction via online dynamic fuzzy neural networks. Engineering Applications of Artificial Intelligence,35, 105-113.

23. Pan, Y., Yu, H. (2016). Biomimetic hybrid feedback feedforward neural-network learning control. IEEE Transactions on Neural Networks and Learning Systems, 28 (6), 1481-1487.

24. Pratama, M., Dimla, E., Lughofer, E. (2017). Metacognitive Learning Approach for Online Tool Condition Monitoring. Journal of Intelligent Manufacturing, 2017.

25. Pratama, M., Lughofer, E., Anavatti, S., Lim, C. P. (2017). Data driven modelling based on recurrent interval-valued metacognitive scaffolding fuzzy neural network. Neurocomputing, 2017.

26. Pratama, M., Lughofer, E., Lim, C. P., Rahayu, W., Dillon, T., Budiyono, A. (2017). pClass+: A novel evolving semisupervised classifier. International Journal of Fuzzy Systems, DOI: 10.1007/s40815016-0236-3.

27. Shanbhag, S. S., Udupi, G. R., Patil, K. M., \& Ranganath, K. (2014). Quantitative 
analysis of diffusion weighted MR images of brain tumor using signal intensity gradient technique. Journal of medical engineering, 2014.

28. Son, L.H., Linh, N. D., \& Long, H. V. (2014). A lossless DEM compression for fast retrieval method using fuzzy clustering and MANFIS neural network. Engineering Applications of Artificial Intelligence, 29, 33-42.

29. Torabi, A. J., Er, M. J., Li, X., Lim, B. S. (2016). Sequential fuzzy clustering based dynamic fuzzy neural network for fault diagnosis and prognosis. Neurocomputing, vol. 196, pp. 31-41.

30. UCI 2015“Heart Attack dataset,” Available at: https://archive.ics.uci.edu/ml/datasets/Heart +Disease(Accessed on: September 15, 2015).

31. Vanini, Z. S., Khorasani, K., \& Meskin, N. (2014). Fault detection and isolation of a dual spool gas turbine engine using dynamic neural networks and multiple model approach. Information Sciences, 259, 234251.

32. Walczak, S. (2018). Artificial neural networks. In Encyclopedia of Information Science and Technology, Fourth Edition (pp. 120-131). IGI Global.

33. Witten, I. H., Frank, E., Hall, M. A., \& Pal, C. J. (2016). Data Mining: Practical machine learning tools and techniques. Morgan Kaufmann.

34. Wu, D., Pigou, L., Kindermans, P. J., Le, N. D. H., Shao, L., Dambre, J., \& Odobez, J. M. (2016). Deep dynamic neural networks for multimodal gesture segmentation and recognition. IEEE transactions on pattern analysis and machine intelligence, 38(8), 1583-1597. 